\title{
Fatores associados à qualidade de vida de idosos que frequentam uma unidade de saúde do Distrito Federal
}

\author{
Factors associated with quality of life of elderly attending a health unit in the Federal District, Brazil
}

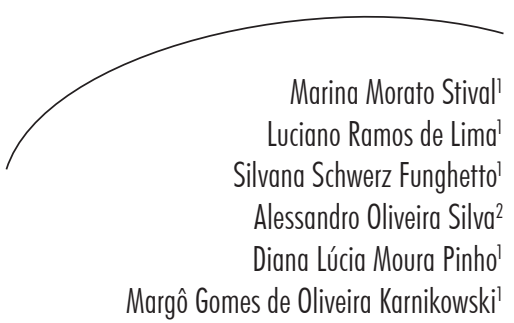

\section{Resumo}

Objetivo: Identificar os fatores associados à qualidade de vida de idosos que frequentam uma unidade de saúde de Ceilândia-DF. Método: Pesquisa de abordagem quantitativa do tipo descritiva com delineamento transversal, realizada com 277 idosos, por meio de entrevista para investigação das variáveis demográficas, socioeconômicas, clínicas e aplicação do WHOQOL-bref, com análise estatística descritiva. Resultados: Os idosos avaliados demonstraram melhor qualidade de vida no domínio "relações sociais", seguido do "psicológico", "físico" e, por último, "meio ambiente". Conclusões: Os fatores significativamente associados à qualidade de vida neste estudo foram: doença, tabagismo, atividade física, alteração visual e história de queda. Os problemas/barreiras conhecidos neste estudo podem direcionar os profissionais de saúde que atuam na atenção primária. A assistência direcionada poderá permitir uma relação de confiança entre o profissional de saúde e o idoso, além de auxiliá-los a resolver os problemas/ barreiras que estão afetando sua qualidade de vida.

\section{Abstract}

Objective: To identify factors associated with quality of life of elderly attending a Health Unit in Ceilandia, Federal District, Brazil. Method: Quantitative approach with descriptive cross-sectional design conducted with 277 elderly through interviews to investigate demographic, socioeconomic, and clinical variables and application of the WHOQOL-bref, with descriptive statistical analysis. Results: The elderly showed better quality of life in the domain "social relations", followed by "psychological", "physical" and finally "environment". Conclusions: Factors significantly associated with quality of life in this study were: disease, smoking, physical activity, visual and history of falls. The problems / barriers known in this study can guide the health professionals who work at primary health care. The targeted assistance may enable a trusting relationship between the health professional and the elderly as well as help them to solve the problems / barriers that affect their quality of life.

\footnotetext{
Faculdade de Ceilândia. Universidade de Brasília. Brasília, DF, Brasil.

2 Programa de Pós-graduação em Gerontologia. Universidade Católica de Brasília. Brasília, DF, Brasil.
}

Palavras-chave: Qualidade de Vida. Idoso. Estilo de Vida. Saúde do Idoso.

Descriptors: Quality of Life. Elderly. Life Style. Health of the Elderly. 


\section{INTRODUÇÃO}

Nos últimos anos, tem-se observado uma transição demográfica que se reflete numa transição epidemiológica no Brasil, determinando uma nova realidade social, que implica grandes necessidades de investigações no sentido de contribuir para uma melhor qualidade de vida (QV) do idoso. ${ }^{1,2} \mathrm{~A}$ percepção dos idosos sobre sua saúde interfere na longevidade e acarreta melhor QV aos mesmos. Os idosos que relataram excelente saúde aos 70 anos de idade obtiveram uma expectativa de vida quatro anos maior em relação àqueles que consideraram sua saúde pobre. ${ }^{3}$

Neste sentido, a QV é considerada um conceito amplo, complexo e com diferentes interpretações. ${ }^{4}$ A qualidade de vida e a percepção de bem-estar de uma pessoa derivam de sua avaliação do quanto realizou, daquilo que idealiza como importante para uma boa vida e de seu grau de satisfação com o que foi possível concretizar até aquele momento. Sendo assim, a qualidade de vida do idoso não é simplesmente a cura ou prevenção de uma doença, mas também o bem-estar psicológico de um indivíduo. ${ }^{5}$

Observa-se também que a QV varia de indivíduo para indivíduo e pode sofrer alterações durante a vida, mostrando que são vários os fatores que identificam a QV para as pessoas, e que estes fatores combinados resultam numa rede de fenômenos e situações. ${ }^{6}$

Uma grande variedade de fatores é válida para a melhoria na saúde do idoso e, consequentemente, a promoção da qualidade de vida, incluindo avanço em tecnologias médicas; mudanças no comportamento pessoal, como interrupção do tabagismo, moderação do consumo de sal e lipídeos; atenção especial para o manejo da dor; e diminuição da exposição às doenças infecciosas, além de apoio social.

Com toda essa diversidade de conceitos sobre QV, pode-se perceber que não é possível chegar a um único consenso, mas observa-se que em diversos deles a QV está na percepção que cada indivíduo posiciona sua vida em um amplo conceito. Ter uma avaliação da QV abrange múltiplos critérios de natureza biológica, psicológica e socioestrutural, pois vários são os determinantes da QV, principalmente na velhice, como saúde, satisfação, produtividade, família e em relação à sociedade. ${ }^{8}$ Neste sentido, houve na última década a criação de instrumentos que avaliasse a qualidade de vida. A maioria deles foi desenvolvida nos Estados Unidos e com crescente interesse em traduzi-los para aplicação em outras culturas.

A busca de um instrumento que avaliasse QV dentro de uma perspectiva genuinamente internacional fez com que a Organização Mundial da Saúde organizasse um projeto colaborativo multicêntrico: o WHOQOL GROUP, que define QV como a percepção do indivíduo de sua posição de vida no contexto da cultura e sistema de valores nos quais ele vive e em relação a seus objetivos, expectativas, padrões e preocupações.?

Diante da diversidade de definições, este trabalho elegeu uma definição que se fez presente em diversos trabalhos pesquisados durante $\mathrm{O}$ levantamento bibliográfico: para o idoso, a QV tem importância fundamental, como a saúde; ou seja, um idoso, estando saudável, significa uma boa QV. O idoso deve ter conhecimento e participar do que será melhor para ele, pois a QV é um fator pessoal. Os estudos sobre as condições que permitem ao idoso uma boa QV revestem-se de grande valor científico e social. ${ }^{5,10}$

Estudos sobre qualidade de vida têmse mostrado importantes em muitas áreas. Conhecer a qualidade de vida do idoso revela-se importante e necessário para nortear condutas, tratamentos e políticas, uma vez que a avaliação seja multidimensional. Fica, portanto, cada vez mais evidente a contribuição que estudos avaliando a qualidade de vida do idoso podem proporcionar na melhoria da atenção ao idoso. Além disso, essas pesquisas podem contribuir ao destacar a importância de os profissionais de saúde conhecerem a qualidade de vida do idoso com vistas a promover bem-estar físico, mental e social, a fim de resultar em melhor independência e autonomia do idoso. 
Assim, este estudo objetivou identificar os fatores associados à qualidade de vida de idosos que frequentam uma unidade de saúde de Ceilândia-DF.

\section{MÉTODOS}

Trata-se de uma pesquisa de abordagem quantitativa do tipo descritiva com delineamento transversal, realizada numa unidade de saúde de Ceilândia-DF. A amostra, por conveniência, foi composta por idosos, de ambos os sexos, com idade igual ou acima de 60 anos e que procuravam atendimento na unidade de saúde de CeilândiaDF, escolhida para realização da pesquisa no período de fevereiro a abril de 2011.

Os idosos foram abordados no momento de chegada à unidade de saúde, e ao aceitarem participar da pesquisa assinavam o Termo de Consentimento Livre e Esclarecido (TCLE). Em seguida, foi realizada uma entrevista em momento único numa sala com privacidade, seguindo com um roteiro semiestruturado para investigação das variáveis demográficas, socioeconômicas e clínicas.

$\mathrm{O}$ instrumento utilizado para avaliar a qualidade de vida foi o WHOQOL-bref, que consta de 26 questões, sendo duas gerais de QV e outras 24 representantes de cada uma das 24 facetas que compõem o instrumento original. Essas 24 questões são agrupadas em quatro domínios: "físico" (sete itens), "psicológico" (seis itens), "relações sociais" (três itens) e "meio ambiente" (oito itens). ${ }^{10,11}$

As facetas dos domínios do WHOQOLbref são: "físico" (dor e desconforto; energia e fadiga; sono e repouso; mobilidade; atividades da vida diária; dependência de medicação ou de tratamentos e capacidade de trabalho); "psicológico" (sentimentos positivos; pensar, aprender, memória e concentração; autoestima; imagem corporal e aparência; sentimentos negativos; espiritualidade/religião/crenças pessoais); "relações sociais" (relações pessoais; suporte-apoio; social e atividade sexual) e "meio ambiente" (segurança física e proteção; ambiente no lar; recursos financeiros; cuidados de saúde e sociais: disponibilidade e qualidade; oportunidades de adquirir novas informações e habilidades; participação em, e oportunidades de recreação/lazer; ambiente físico: poluição/ruído/ trânsito/clima e transporte). ${ }^{10,11}$

As questões do WHOQOL-bref são formuladas para respostas em escalas tipo Likert, incluindo intensidade (nada e extremamente), capacidade (nada e completamente), frequência (nunca e sempre) e avaliação (muito insatisfeito, muito satisfeito, muito ruim e muito bom). As pontuações de cada domínio e total são transformadas numa escala de 0 a 100 , e os maiores escores indicam melhor QV. ${ }^{10,11}$

Para análise dos resultados, foi utilizado o programa estatístico Special Package for Social Sciences (SPSS) versão 16.0. Foi realizada uma análise quantitativa descritiva, de acordo com as instruções do instrumento, e calculados os escores totais de cada respondente, bem como os valores médios de cada um dos fatores, domínios e dimensões. O teste Mann-Whitnney foi utilizado para comparação de médias entre dois grupos e o teste de Kruskal-Wallis no caso de três níveis ou mais. A avaliação da consistência interna do WHOQOL-bref foi avaliada por meio do coeficiente Alfa de Cronbach. O nível de significância utilizado foi de 5\%.

Esta pesquisa foi aprovada pelo Comitê de Ética em Pesquisa da Secretaria do Estado de Saúde do Distrito Federal (SES/DF), sob parecer no 194/2010.

\section{RESULTADOS}

Dos 277 idosos estudados, a maioria era do sexo feminino (63,5\%), com idade entre 60 e 65 anos (45,8\%), aposentados (53,4\%), casados $(54,9 \%)$, com ensino fundamental $(73,3 \%)$, renda de até um salário mínino $(59,9 \%)$ e não morava sozinho $(83,4 \%)$, segundo a tabela 1.

O domínio em que os idosos demostraram melhor QV foi "relações sociais". Evidenciouse, também, que no domínio "meio ambiente" os escores de QV aumentam com o decorrer da idade $(\mathrm{p}=0,006)$. 
As demais variáveis demográficas e socioeconômicas não foram significativamente associadas aos escores de qualidade de vida. No entanto, verifica-se que aqueles idosos que afirmaram ter ensino superior, renda superior a quatro salários mínimos e os que não moravam sozinhos apresentaram maiores escores de QV nos quatro domínios (tabela 1).

Tabela 1. Comparação das médias dos escores dos domínios do Whoqol-bref, de acordo com características demográficas e socioeconômicas. Brasília-DF, 2011.

\begin{tabular}{|c|c|c|c|c|c|}
\hline & \multirow[b]{2}{*}{ n $(\%)$} & \multicolumn{4}{|c|}{ Média dos domínios } \\
\hline & & Físico & Psicológico & Relações sociais & Meio ambiente \\
\hline \multicolumn{6}{|l|}{ Sexo } \\
\hline Masculino & $101(36,5)$ & 74,76 & 78,34 & 80,99 & 68,26 \\
\hline Feminino & $176(63,5)$ & 72,35 & 78,95 & 80,87 & 67,74 \\
\hline Valor $\mathrm{p}^{*}$ & & 0,194 & 0,717 & 0,953 & 0,745 \\
\hline \multicolumn{6}{|l|}{ Idade (anos) } \\
\hline 60 a 65 & $127(45,8)$ & 71,91 & 77,03 & 81,04 & 65,31 \\
\hline 66 a 70 & $78(28,2)$ & 74,04 & 79,10 & 81,28 & 68,84 \\
\hline 71 a 75 & $43(15,5)$ & 74,68 & 81,00 & 81,39 & 71,50 \\
\hline$>75$ & $29(10,5)$ & 74,67 & 81,83 & 78,62 & 71,63 \\
\hline Valor $\mathrm{p}^{* *}$ & & 0,514 & 0,199 & 0,973 & 0,006 \\
\hline \multicolumn{6}{|l|}{ Aposentado } \\
\hline $\operatorname{Sim}$ & $148(53,4)$ & 73,60 & 77,92 & 79,68 & 68,71 \\
\hline Não & $129(46,6)$ & 72,80 & 79,66 & 82,32 & 67,03 \\
\hline Valor $\mathrm{p}^{*}$ & & 0,653 & 0,284 & 0,174 & 0,280 \\
\hline \multicolumn{6}{|l|}{ Estado civil } \\
\hline Casado & $152(54,9)$ & 74,92 & 78,83 & 81,97 & 68,56 \\
\hline Solteiro & $34(12,3)$ & 72,85 & 78,92 & 78,62 & 64,77 \\
\hline Divorciado & $32(11,6)$ & 72,05 & 78,43 & 82,70 & 66,79 \\
\hline Viúvo & $59(21,3)$ & 69,73 & 78,53 & 78,53 & 68,72 \\
\hline Valor $\mathrm{p}^{* *}$ & & 0,068 & 0,957 & 0,340 & 0,369 \\
\hline \multicolumn{6}{|l|}{ Escolaridade } \\
\hline Analfabeto & $40(14,4)$ & 70,42 & 76,91 & 81,00 & 67,87 \\
\hline Ensino fundamental & $203(73,3)$ & 73,56 & 79,12 & 80,88 & 67,70 \\
\hline Ensino médio & $30(10,8)$ & 73,61 & 77,55 & 80,88 & 68,41 \\
\hline Ensino superior & $4(1,4)$ & 81,42 & 85,83 & 81,66 & 76,25 \\
\hline Valor $\mathrm{p}^{* *}$ & & 0,379 & 0,586 & 0,818 & 0,648 \\
\hline \multicolumn{6}{|l|}{ Renda } \\
\hline Até $1 \mathrm{SM}$ & $166(59,9)$ & 72,34 & 78,81 & 81,72 & 67,80 \\
\hline De 1 a $3 \mathrm{SM}$ & $87(31,4)$ & 73,87 & 78,31 & 78,23 & 67,72 \\
\hline De 4 a 6 SM & $24(8,7)$ & 77,02 & 79,72 & 85,00 & 69,58 \\
\hline Valor $\mathrm{p}^{* *}$ & & 0,397 & 0,942 & 0,198 & 0,890 \\
\hline \multicolumn{6}{|l|}{ Mora sozinho } \\
\hline Sim & $46(16,6)$ & 70,31 & 75,57 & 77,24 & 64,89 \\
\hline Não & $231(83,4)$ & 73,81 & 79,36 & 81,64 & 68,53 \\
\hline Valor $\mathrm{p}^{*}$ & & 0,144 & 0,081 & 0,091 & 0,080 \\
\hline
\end{tabular}

*TesteMann-Whitnney; **Kruskal-Wallis; SM= salário mínimo. 
Quanto às características clínicas, observouse que a hipertensão arterial foi a doença mais prevalente, sendo encontrada isoladamente $(42,2 \%)$ e associada à diabetes mellitus $(20,6 \%)$. Dos 277 idosos avaliados, 88,1\% afirmaram não ser tabagista, 90,3\% não eram etilistas, $61,7 \%$ não praticavam atividade física, 70,8\% possuíam alteração visual e faziam uso de óculos, 31,8\% com alterações auditivas e 46,6\% relataram já ter sofrido pelo menos um episódio de queda (tabela 2).

Em relação à presença de doenças, evidenciou-se que aqueles idosos que não tinham doença apresentaram melhores escores de QV nos domínios "físico" ( $\mathrm{p}<0,001)$, "psicológico" $(p=0,026)$ e "meio ambiente" ( $p=0,009) . O$ tabagismo também foi significativamente associado à QV nos domínios "psicológico" $(p=0,006)$, "relações sociais" $(p=0,016)$ e "meio ambiente" ( $p=0,041)$, sendo que os que afirmaram não fumar demonstraram melhor QV nesses domínios. Os idosos que realizavam atividade física também apresentaram melhor $\mathrm{QV}$, porém uma associação significativa foi observada nos domínios "físico" $(\mathrm{p}=0,001)$ e "meio ambiente" $(p=0,001)$, conforme a tabela 2 .

Os idosos que não apresentaram alteração visual também apresentaram melhor QV nos quatro domínios, sendo que no "físico" esta associação ficou evidente ( $\mathrm{p}=0,047)$. Nota-se que os idosos com história de queda demonstraram pior QV, e nos domínios "físico" $(\mathrm{p}<0,001)$ e "meio ambiente" $(p=0,002)$, essa influência foi significativa (tabela 2). 
Tabela 2. Comparação das médias dos escores dos domínios do Whoqol-bref, de acordo com características clínicas e hábitos de vida. Brasília-DF, 2011.

\begin{tabular}{|c|c|c|c|c|c|}
\hline \multirow{3}{*}{ Doença } & \multirow[b]{2}{*}{$\mathrm{n}(\%)$} & \multicolumn{4}{|c|}{ Média dos domínios } \\
\hline & & \multirow[t]{2}{*}{ Físico } & \multirow[t]{2}{*}{ Psicológico } & \multirow[t]{2}{*}{ Relações sociais } & \multirow[t]{2}{*}{ Meio ambiente } \\
\hline & & & & & \\
\hline Nenhuma & $36(13,0)$ & 86,19 & 81,85 & 80,92 & 71,94 \\
\hline HAS & $117(42,2)$ & 74,24 & 79,74 & 82,16 & 69,82 \\
\hline $\mathrm{DM}$ & $22(7,9)$ & 64,8 & 70,15 & 77,57 & 62,84 \\
\hline HAS e DM & $57(20,6)$ & 69,24 & 76,54 & 81,98 & 65,52 \\
\hline Outras & $45(16,2)$ & 69,39 & 80,59 & 77,92 & 65,33 \\
\hline Valor $\mathrm{p}^{* *}$ & & $<0,001$ & 0,026 & 0,580 & 0,009 \\
\hline \multicolumn{6}{|l|}{ Tabagista } \\
\hline $\operatorname{Sim}$ & $33(11,9)$ & 71,68 & 72,92 & 74,14 & 63,86 \\
\hline Não & $244(88,1)$ & 73,44 & 79,52 & 81,03 & 68,42 \\
\hline Valor $\mathrm{p}^{*}$ & & 0,528 & 0,006 & 0,016 & 0,041 \\
\hline \multicolumn{6}{|l|}{ Etilista } \\
\hline Sim & $27(9,7)$ & 76,08 & 80,37 & 80,00 & 69,72 \\
\hline Não & $250(90,3)$ & 72,92 & 78,56 & 81,01 & 67,74 \\
\hline Valor $\mathrm{p}^{*}$ & & 0,294 & 0,507 & 0,757 & 0,449 \\
\hline \multicolumn{6}{|c|}{ Prática atividade física } \\
\hline Sim & $106(38,3)$ & 77,84 & 80,69 & 80,88 & 71,06 \\
\hline Não & $171(61,7)$ & 70,37 & 77,52 & 80,93 & 65,99 \\
\hline Valor $\mathrm{p}^{*}$ & & 0,001 & 0,056 & 0,978 & 0,001 \\
\hline \multicolumn{6}{|l|}{ Alteração visual } \\
\hline $\operatorname{Sim}$ & $196(70,8)$ & 72,09 & 77,87 & 80,71 & 67,55 \\
\hline Não & $81(29,2)$ & 75,99 & 80,82 & 81,39 & 68,85 \\
\hline Valor $\mathrm{p}^{*}$ & & 0,047 & 0,096 & 0,748 & 0,444 \\
\hline \multicolumn{6}{|l|}{ Alteração auditiva } \\
\hline $\operatorname{Sim}$ & $88(31,8)$ & 70,86 & 77,99 & 80,15 & 67,38 \\
\hline Não & $189(68,2)$ & 74,33 & 79,08 & 81,26 & 68,18 \\
\hline Valor p* & & 0,070 & 0,530 & 0,591 & 0,631 \\
\hline \multicolumn{6}{|l|}{ História de queda } \\
\hline Sim & $129(46,6)$ & 69,32 & 77,95 & 80,46 & 65,38 \\
\hline Não & $148(53,4)$ & 76,63 & 79,41 & 81,30 & 70,15 \\
\hline Valor $\mathrm{p}^{*}$ & & $<0,001$ & 0,369 & 0,665 & 0,002 \\
\hline
\end{tabular}

*TesteMann-Whitnney; **Kruskal-Wallis; HAS= hipertensão arterial; DM= diabetes mellitus. 
Sabe-se que na avaliação dos domínios do WHOQOL-bref, quanto mais próxima a média for de 100, melhor será a qualidade de vida. No geral, evidenciou-se que os idosos estudados apresentaram melhor QV no domínio "relações sociais" $(\mathrm{m}=80,91)$, seguido do domínio "psicológico" $(\mathrm{m}=78,73)$, "físico" $(\mathrm{m}=73,23)$ e, por último, "meio ambiente" ( $\mathrm{m}=67,93)$, conforme mostra a tabela 3.
A avaliação da consistência interna do WHOQOL-bref por meio do coeficiente Alfa de Cronbach mostrou homogeneidade dos domínios, porém com valores limítrofes ao considerado aceitável e sendo constatado menor valor no domínio "psicológico" (tabela 3).

Tabela 3. Medidas descritivas e coeficiente de fidedignidade de Alfa de Cronbach dos domínios do Whoqol-bref. Brasília-DF, 2011.

\begin{tabular}{lccccc}
\hline & $\mathrm{m}$ & $\mathrm{dp}$ & Mín. & Máx. & Cronbach \\
\hline Domínio I - Físico & 73,23 & 14,85 & 28,57 & 100,00 & 0,746 \\
Domínio II - Psicológico & 78,73 & 13,42 & 40,00 & 100,00 & 0,683 \\
Domínio III - Relações sociais & 80,91 & 16,10 & 20,00 & 100,00 & 0,740 \\
Domínio IV - Meio ambiente & 67,93 & 12,89 & 32,50 & 100,00 & 0,717 \\
\hline
\end{tabular}

m= média; $\mathrm{dp}=$ desvio-padrão; Mín.= mínimo; Máx.= máximo.

\section{DISCUSSÃO}

Os idosos avaliados demonstraram melhor qualidade de vida no domínio "relações sociais", seguido do "psicológico", "físico" e, por último, "meio ambiente". Resultados semelhantes foram observados em outro estudo, que também utilizou o WHOQOL-bref na avaliação de QV em idosos, e os melhores escores dos domínios, de forma decrescente, foram: "social", "físico", "psicológico" e "meio ambiente".12

Sabe-se que o domínio "relações sociais" está relacionado à satisfação do idoso com suas relações pessoais (amigos e familiares), ao suporte e apoio recebido e sua atividade sexual. Outros estudos também evidenciaram melhor QV neste domínio. ${ }^{6,12-15}$
Neste estudo, apesar de a maioria de idosos ser aposentada, pode-se atribuir esta satisfação com suas relações sociais ao fato de a maioria dos idosos desta comunidade participarem de atividades de lazer em praças, grupos de convivência e atividades na igreja, permitindo, portanto, maior convivência com amigos.

Em outro estudo realizado com idosos em Porto Alegre-RS, evidenciou-se que alguns fatores como as relações familiares, de amizade e suporte psicossocial estão associadas ao envelhecimento bem-sucedido. ${ }^{16}$ Por outro lado, os idosos demonstraram pior QV no domínio "meio ambiente". Este resultado não condiz com o estudo realizado com idosos de VeranópolisRS, no qual este domínio apresentou escores elevados, demonstrando, portanto, boa interação 
dos idosos com o meio ambiente. Em outros estudos também têm sido observadas fortes associações entre o aspecto ambiental e a QV de idosos, resultando em sentimento de segurança, interação social, independência e bem-estar emocional. ${ }^{16,17}$

Os piores escores de QV evidenciados no domínio "meio ambiente" podem estar relacionados ao fato de os idosos não se sentirem seguros e protegidos em seu ambiente doméstico, principalmente por se tratar de uma região com elevados índices de violência, e também pela maioria de os idosos entrevistados apresentarem baixa renda (até um salário mínimo) e viver em regiões com ambientes precários, no que se refere a ruas e asfaltos. Geralmente idosos que vivem em regiões inseguras não saem sozinhos e ficam mais suscetíveis a isolamento e depressão, o que pode influenciar na $\mathrm{QV} .{ }^{12}$

Esses problemas foram mais relatados por idosos jovens, uma vez que neste estudo a idade foi associada ao domínio "meio ambiente", pois os escores de QV aumentaram no decorrer da idade. É importante considerar a influência da idade na QV de idosos, porém os resultados deste estudo não corroboram os de outras pesquisas, nas quais geralmente se observa uma queda da QV com o decorrer da idade..$^{12,14,18}$ Esta discordância pode ser justificada devido ao fato de a maioria da amostra estudada ter idade entre 60 e 65 anos. Os fatores significativamente associados à QV neste estudo foram: doença, tabagismo, atividade física, alteração visual e história de queda.

Os idosos que não apresentaram doença demonstraram melhor QV nos domínios "f́́sico", "psicológico" e "meio ambiente". Pacientes com diabetes mellitus foram os que apresentaram os piores escores de QV nestes domínios. Em estudo realizado em UberabaMG com 1.378 idosos com e sem hipertensão arterial, evidenciou-se que o grupo de idosos sem a doença apresentou melhores escores de QV e o grupo de idosos portadores da doença apresentou os piores escores de QV nos domínios "físico" e "meio ambiente".

Observa-se que os idosos sem doenças apresentam melhor QV no domínio "físico". Em outro estudo, também ficou demonstrada tal associação entre presença de enfermidades e pior QV neste domínio. ${ }^{20}$

Esse fato pode ser explicado pelo impacto que uma doença, principalmente a diabetes, causa na vida do indivíduo, podendo gerar desconforto e até mesmo dor, influenciando assim negativamente suas atividades do cotidiano. Ainda, a presença de comorbidades também pode acarretar a necessidade de usar medicações e de maior acesso aos serviços de saúde, levando o idoso a ficar dependente de serviços públicos por se tratar de idosos de baixa renda.

Neste estudo também foram observados maiores escores nos domínios "psicológico", "relações sociais" e "meio ambiente" de idosos que não eram tabagistas, evidenciando, portanto, associação entre tabagismo e QV. Em outros estudos, também foi encontrada esta associação, porém na maioria deles o domínio "físico" também esteve presente..$^{20,21}$

Geralmente observa-se que o idoso, por fumar há muitos anos, apresenta mais resistência em abandonar o vício, principalmente por considerar que os danos do cigarro já acometeram seu organismo. É demonstrado também, em alguns estudos, que o tabaco pode ser usado pelo idoso para aumentar sua autoestima, relaxamento e até alívio da dor. ${ }^{20}$ Apesar disso, ressalta-se que é evidente que o abandono do hábito de fumar pode representar melhora significativa na QV do idoso, além de diminuir o risco de doenças cardiovasculares e pulmonares.

Os idosos que realizam atividade física apresentaram melhor QV nos domínios "físico" e "meio ambiente". Estes resultados corroboram os encontrados em outra pesquisa. ${ }^{21} \mathrm{E}$ importante 
destacar o efeito positivo da atividade física, tanto na saúde física como mental do idoso. Neste sentido, foi realizado estudo com mulheres idosas, que encontrou esta associação, ou seja, as idosas que praticavam atividade física apresentaram melhor QV nos domínios "físico" e "psicológico".22

Neste estudo 38,3\% dos idosos praticavam atividade física. Com esta realidade, sugere-se um processo de intervenção, a fim de aumentar a prática de atividade física dos idosos como uma estratégia eficaz no aumento dos escores de QV, principalmente no domínio "físico", por melhorar capacidade funcional, dor e até mesmo aumentar a disposição dos idosos para atividades do cotidiano.

Outros fatores associados à QV dos idosos neste estudo foram a alteração visual, pois foi evidenciada melhor QV no domínio "físico" em idosos que não apresentaram alterações e também em idosos com história de queda que apresentaram pior QV nos domínios "físico" e "meio ambiente". Sabe-se que as alterações visuais relacionadas à idade têm aumentado devido ao envelhecimento populacional e, além disso, as alterações podem ser consideradas um fator contributivo para as quedas nos idosos. ${ }^{23,24}$ Entende-se que os idosos com déficit visual e com histórico de quedas tendem a diminuir suas atividades do cotidiano, que é um dos itens avaliados no domínio "físico".

Em pesquisa realizada objetivando estudar o impacto das quedas na qualidade de vida do idoso, ficou evidente que tal evento tem consequências físicas, funcionais e psicossociais na vida do idoso. ${ }^{25}$ Além disso, em outro estudo também foi demonstrada a influência da queda na qualidade de vida do idoso, porém mais significativa no domínio "psicológico". ${ }^{26}$

Contraditoriamente, neste estudo a história de queda teve mais influência nos domínios "físico" e "meio ambiente". Em geral, o idoso que teve queda uma vez apresenta alguma fratura ou até mesmo medo de cair novamente, limitando, portanto, suas atividades e interação com o ambiente. Com isso, percebe-se que a queda pode ter impacto negativo na QV dos idosos, podendo incluir morbidades, diminuição da capacidade funcional e aumento da utilização dos serviços públicos de saúde. Além disso, pode causar desconforto, dores, incapacidade e medo de cair novamente. ${ }^{26}$

Este estudo teve como limitações não ter analisado a quantidade de medicamentos domiciliares utilizados pelo idoso $e$ as complicações da diabetes mellitus, que podem ter impacto na sua qualidade de vida. Os valores limítrofes de consistência interna do instrumento podem ser justificados pela dificuldade do idoso no entendimento das questões do instrumento de coleta de dados.

\section{CONCLUSÃO}

A análise estatística proposta no estudo foi considerada satisfatória, pois foi possível evidenciar os fatores significativamente associados à qualidade de vida do idoso: doença, tabagismo, atividade física, alteração visual e história de queda. Foi demonstrado que os idosos apresentam melhor qualidade de vida no domínio "relações sociais", seguido do "psicológico", "físico" e, por último, "meio ambiente".

Os fatores identificados neste estudo podem permitir aos profissionais de saúde repensar estratégias de saúde voltadas, principalmente, para os domínios afetados na qualidade de vida do idoso, sobretudo aqueles que trabalham em unidades de saúde que desenvolvem atividades preventivas, assistenciais, educativas e de atenção à saúde do idoso.

A assistência direcionada poderá permitir uma relação de confiança entre o profissional de saúde e o idoso, além de auxiliá-los a resolver os problemas/barreiras que estão afetando sua qualidade de vida. 


\section{REFERÊNCIAS}

1. Instituto Brasileiro de Geografia e Estatística. Sinopse do censo demográfico de 2010 [Internet]. Rio de Janeiro: IBGE; 2011 [acesso em 1 Jun 2011]. Disponível em: http://www.ibge.gov.br/home/ estatistica/populacao/censo2010/sinopse.pdf

2. Araujo LF, Coutinho MPL, Santos MFS. O idoso nas instituições gerontológicas: um estudo na perspectiva das representações sociais. Psicol Soc [Internet] 2006 [acesso em 14 jan 2012];18(2):89-98. Disponível em: http://www.scielo.br/scielo.php?pid=S0102$71822006000200012 \&$ script $=$ sci_abstract $\&$ tlng $=$ pt

3. Lubitz J, Cai L, Kramarow E, Lentzner H. Health, life expectancy, and health care spending among the elderly. N Engl J Med [Internet] 2003 [acesso em 10 fev 2012];349(11):1048-55. Disponível em: http:// www.nejm.org/doi/full/10.1056/NEJMsa020614

4. Inouye K, Pedrazzani ES. Nível de instrução, status socioeconômico e avaliação de algumas dimensões da qualidade de vida de octogenários. Rev Latino-Am Enfermagem [Internet] 2007 [acesso em 14 Out 2011];15 n.spe:742-7. Disponível em: http://www.scielo.br/scielo.php?pid=S0104$11692007000700005 \&$ script $=$ sci_arttext\&tlng=pt

5. Paschoal SMP. Qualidade de vida do idoso: elaboração de um instrumento que privilegia sua opinião [Dissertação]. São Paulo: Universidade de São Paulo, Programa de Pós-Graduação em Medicina; 2000.

6. Alexandre TS, Cordeiro RC, Ramos LR. Factors associated to quality os life in active elderly. Rev Saúde Pública [Internet] 2009 [acesso em 04 Fev 2012]; 43(4):613-21. Disponível em: http:// www.scielo.br/scielo.php?script=sci_arttext\&pid =S0034-89102009000400007

7. Rice DP, Fineman N. Economic implications of increased longevity in the United States. Ann Rev Public Health [Internet] 2004 [acesso em 10 Dez 2011];25:457-73. Disponível em: http://www. annualreviews.org/doi/full/10.1146/annurev. publhealth.25.101802.123054

8. Fleck MPA. O Instrumento de Avaliação da Qualidade de Vida da Organização Mundial da Saúde (WHOQOL-100): características e perspectivas. Ciênc Saúde Coletiva [Internet] 2000 [acesso em 09 Dez 2011];5(1):33-8. Disponível em: http://www. scielo.br/pdf/csc/v5n1/7077.pdf

9. Universidade Federal do Rio Grande Do Sul, Famed/ Hcpa, Grupo de Estudos em Qualidade de Vida. Versão em português dos instrumentos de avaliação de qualidade de vida (WHOQOL) 1998 [Internet].
Porto Alegre: Departamento de Psiquiatria e Medicina Legal; 1998 [acesso em 09 Dez 2011]. Disponível: http://www.ufrgs.br/psiq/WHOQOL.html

10. Fleck MPA, Chachamovich E, Trentini C. Projeto WHOQOL-OLD: método e resultados de grupos focais no Brasil. Rev Saúde Pública [Internet] 2003 [acesso em 25 Jan 2012];37(6):793-9. Disponível em: http://www.scielo.br/scielo.php?pid=S003489102003000600016\&script=sci_arttext

11. World Health Organization, WHOQOL GROUP. Development of the WHOQOL-BREF quality of life Assessment. Psychol Med [Internet] 1998 [acesso em 10 Fev 2012];28(3):551-8. Disponível em: http://www. ncbi.nlm.nih.gov/pubmed/9626712

12. Pereira RJ, Cotta RM, Franceschini SCC. Contribuição dos domínios físico, social, psicológico e ambiental para a qualidade de vida global de idosos. Rev Psiquiatr [Internet] 2006 [acesso em 08 mar 2012];28(1):27-38. Disponível em: http://www.scielo.br/scielo.php?pid=S010181082006000100005\&script=sci_arttext

13. Freitas VFFS. Qualidade de vida no município de Herval. Rev Enferm Saúde [Internet] 2011 [acesso em 24 Nov 2011];1(1):156-63. Disponível em:http://www. ufpel.edu.br/revistas/index.php/enfermagemesaude/ article/viewPDFInterstitial/54/39

14. Farenza WP, Argimon IL, Moriguchi E, Portuguez MW. Qualidade de vida em um grupo de idosos de Veranópolis. Rev Kairos [Internet] 2007 [acesso em 10 Set 2011];10(2):255-43. Disponível em: http:// www.scielo.br/scielo.php?script=sci_arttext\&pid =S0034-89102009000400007

15. Tavares DMS, Dias FA. Capacidade funcional, morbidades e qualidade de vida de idosos. Texto \& Contexto Enferm [Internet] 2012 [acesso em 22 Set 2011];21(1):112-20. Disponível em: http://www.scielo.br/scielo.php?pid=S010407072012000100013\&script=sci_arttext

16. Moraes JFD, Souza VBA. Fatores associados ao envelhecimento bem-sucedido de idosos socialmente ativos da região metropolitana de Porto Alegre. Rev Bras Psiquiatr [Internet] 2005 [acesso em 15 Mai 2012];27(4):302-8. Disponível em: http://www.scielo.br/scielo.php?pid=S151644462005000400009\&script=sci_arttext

17. Hwang H, Liang W, Chiu Y, Lin M. Suitability of the WHOQOL-Bref for community-dwelling older people in Taiwan. Age Ageing [Internet] 2003 [acesso em 29 Fev 2012];32(6):593-600. Disponível em: http://ageing. oxfordjournals.org/content/32/6/593.full.pdf 
18. Garcia EL, Banegas JR, Perez-Regadera AG, Cabrera RH, Rodriguez-Artalejo F. Social network and health related quality of life in older adults: a populationbased study in Spain. Qual Life Res [Internet] 2005 [acesso em 22 Nov 2011];14(2):511-20. Disponível em: http://www.ncbi.nlm.nih.gov/pubmed/15892440

19. Tavares DMS, Martins NPF, Dias FA, Diniz MA. Qualidade de vida de idosos com e sem hipertensão arterial. Rev Eletr Enf [Internet] 2011 [acesso em 02 Mar 2012];13(2):211-9. Disponível em: http://www. fen.ufg.br/revista/v13/n2/v13n2a07.htm

20. Pereira RJ, Cotta RM, Franceschini SCC, Ribeiro RCL, Sampaio RF, Priore SE, et al. Influência de fatores sociossanitários na qualidade de vida dos idosos de um município do Sudeste do Brasil. Ciênc Saúde Coletiva [Internet] 2011 [acesso em 22 Jan 2012];16(6):2907-17. Disponível em: http://www.scielo.br/scielo.php?pid=S1413$81232011000600028 \&$ script $=$ sci_arttext

21. Lima MG, Barros MBA, Cesar CLG, Goldbaum M, Carandina L, Alves MCGP. Health-related behavior and quality of life among the elderly: a populationbased study. Rev Saúde Pública [Internet] 2011 [acesso em 02 Ago 2012];45(3):485-93. Disponível em: http://www.scielo.br/scielo.php?script=sci_arttext\&p id $=$ S0034-89102011000300006
22. Mazo, GZ. Atividade física e qualidade de vida de mulheres idosas [Tese]. Porto, PT: Faculdade de Ciências do Desporto e de Educação Física da Universidade do Porto; 2000.

23. Macedo BG, Pereira LSM, Gomes PF, Silva JP, Castro ANV. Impacto das alterações visuais nas quedas, desempenho funcional, controle postural e no equilibrio dos idosos: uma revisão de literatura. Rev Bras Geriatr Gerontol [Internet] 2008 [acesso em 11 Abr 2012];11(3)419-32. Disponível em: http://www. crde-unati.uerj.br/img_tse/v11n3/capitulo9.pdf

24. Lord SR. Visual risk factors for falls in older people. Age Ageing [Internet] 2006 [acesso em $22 \mathrm{Nov}$ 2011];35(Suppl 2):42-45. Disponível em: http://www. ncbi.nlm.nih.gov/pubmed/16926203

25. Lopes RA, Dias RC. O impacto das quedas na qualidade de vida dos idosos. Conscientiae Saúde [Internet] 2010 [acesso em 12 Jun 2012];9(3):504-9. Disponível em: http://redalyc.uaemex.mx/src/inicio/ ArtPdfRed.jsp?iCve=92915180022

26. Ribeiro AP, Souza ER, Atie S, Souza AC, Schilithz AO. A influência das quedas na qualidade de vida dos idosos. Ciênc Saúde Coletiva [Internet] 2008 [acesso em 21 Nov 2011];13(4):1265-73. Disponível em: http://www.scielo.br/scielo.php?pid=S1413$81232008000400023 \&$ script=sci_arttext 